\title{
Bacterial communities in the gut of wild and mass-reared Zeugodacus cucurbitae and Bactrocera dorsalis revealed by metagenomic sequencing
}

\author{
Ashok B. Hadapad ${ }^{1}$, Suresh K. G. Shettigar ${ }^{2}$ and Ramesh S. Hire ${ }^{1,3^{*}}$
}

\begin{abstract}
Background: Insect pests belonging to genus Bactrocera sp. (Diptera: Tephritidae) pose major biotic stress on various fruits and vegetable crops around the world. Zeugodacus and Bactrocera sp. are associated with diverse bacterial communities which play an important role in the fitness of sterile insects. The wild populations of melon fly, Zeugodacus cucurbitae (Coquillett) and Oriental fruit fly, Bactrocera dorsalis (Hendel) were collected from pumpkin and mango fields, respectively. The laboratory populations of Z. cucurbitae and B. dorsalis were mass-reared on bottle gourd and sweet banana, respectively. Bacterial communities present in the gut of wild and mass-reared mature ( $\sim 2$ days old) and newly emerged ( $<1 \mathrm{~h}$ after emergence) male and female adults of $Z$. cucurbitae and $B$. dorsalis were assessed. We used Illumina HiSeq next-generation sequencing of $16 \mathrm{~S} r R N A$ gene to profile the gut bacterial communities of wild and mass-reared mature and newly emerged Z. cucurbitae and B. dorsalis adults.
\end{abstract}

Results: We found diverse bacterial composition in the gut of wild and mass-reared Z. cucurbitae (ZC) and B. dorsalis (BD) with varied relative abundance. Few taxonomic groups were common to both the species. The most dominant phyla in all samples of $Z$. cucurbitae and $B$. dorsalis adults were Actinobacteria, Bacteroidetes, Firmicutes and Proteobacteria. The phylum Proteobacteria occurred more in wild Z. cucurbitae $(\sim 87.72 \%)$ and B. dorsalis ( $\sim 83.87 \%)$ as compared to massreared Z. cucurbitae $(64.15 \%)$ and B. dorsalis ( 80.96\%). Higher relative abundance of Phylum Firmicutes was observed in mass-reared fruit fly than wild adults. Cyanobacteria/Chloroplast and Actinobacteria were also present with very low relative abundance in both wild as well as mass-reared melon fly and Oriental fruit fly. Enterobacteriaceae (61.21\%) was dominant family in the gut of both wild and mass-reared adults. Providencia and Lactococcus were dominant genera with varied relative abundance in wild as well as in mass-reared mature and newly emerged fruit fly adults of both species. Some of the genera like Morganella and Serratia were only detected in mass-reared mature and newly emerged $Z$. cucurbitae and B. dorsalis adults. Principal Coordinate Analysis (PCOA) showed that fruit fly adult samples were grouped based on species and age of the adults while no grouping was observed on the basis of sex of the adult fruit fly.

Conclusions: The gut bacterial communities associated with wild and mass-reared mature and newly emerged adults of $Z$. cucurbitae and B. dorsalis showed variation that depends on species and age of the insects. Understanding the gut microbiota of wild and mass-reared Z. cucurbitae and B. dorsalis using high throughput technology will help to illustrate microbial diversity and this information could be used to develop efficient mass-rearing protocols for successful implementation of sterile insect technique (SIT).

Keywords: Gut bacteria, Metagenomic sequencing, Bactrocera sp., Mass rearing, Bacterial endosymbionts, SIT

\footnotetext{
* Correspondence: rshire@barc.gov.in

'Nuclear Agriculture \& Biotechnology Division, Bhabha Atomic Research

Centre, Trombay, Mumbai 400 085, India

${ }^{3}$ Homi Bhabha National Institute (HBNI), Training School Complex,

Anushaktinagar, Mumbai 400 094, India

Full list of author information is available at the end of the article
}

(C) The Author(s). 2019 Open Access This is an open access article distributed under the terms of the Creative Commons Attribution IGO License (https://creativecommons.org/licenses/by/3.0/igo/) which permits unrestricted use, distribution, and reproduction in any medium, provided appropriate credit to the original author(s) and the source is given. 


\section{Background}

Fruit flies (Diptera: Tephritidae) are the economically important insect pest species and are responsible for damaging agricultural and horticultural crops. Tephritids are distributed in temperate, tropical and subtropical regions of the world [1]. The melon fly, Zeugodacus cucurbitae (Coquillett) and the Oriental fruit fly, Bactrocera dorsalis (Hendel) are the major insect pests of fruits and vegetables across Asia, Africa, Australia, and the South Pacific [1-3]. These fruit flies can cause huge economic losses in India to fruits and vegetables which vary from 30 to $100 \%$ depending upon the crop and season $[2,4]$. Due to their vast adaptability, high reproduction potential and invasion capacity, tephritids have been subject of worldwide pest management programmes. The sterile insect technique (SIT) is an environment friendly, species-specific method of pest control and has been successfully implemented against various insect pests control including fruit flies $[5,6]$. It has been observed that fitness of sterile males is linked with their gut microbiota [7-11]. Irradiation affects the gut microbiota and various strategies have been implemented to augment the gut microbiota using probiotics to regain the fitness of sterile insects [7-11].

Microorganisms inhabiting the intestinal tract of insects play an important role in nutrition, development, survival, resistance to pathogens, and reproduction of the host [8, 12-14]. The gut microbial composition may vary from insect to insect due to their different feeding habits [12, 13]. Different species of bacteria have been isolated and identified from the gut of various fruit fly species including Bactrocera species [15-20] and mainly belong to families Enterobacteriaceae, Bacillaceae, Pseudomonadaceae, Streptococcaceae, Micrococcaceae. Recently, it has been documented in a related species, Ceratitis capitata Wied., that supplementation of adult diet with certain gut bacteria enhances mating competitiveness, longevity, flight ability, improved pupal and adult productivity [8-11]. This will certainly help in successful implementation of SIT programme. Moreover, some gut bacteria and their supernatants have also been found to be promising in attracting fruit fly adults $[18,19,21,22]$. Further, understanding gut bacterial diversity will facilitate in identification of important bacteria and will provide context to the differences in gut microbial communities between populations [20].

Culture dependent approaches have been employed for isolation and characterization of gut microbes in different Tephritidae species and these studies have revealed significant microbial diversity $[8,16,18,19,23-25]$. Molecular approaches such as $16 S$ rRNA gene analysis, DNA fingerprinting, Denaturing gradient gel electrophoresis (DGGE) and oligonucleotide probe-based hybridization techniques are enabling accurate identification of microbial communities of insects $[8,12,13,15,17,19,23,26]$. However, insect gut harbor vast number of unculturable bacteria [27] which play an important role in biology of insects [12]. High throughput technologies with bacterial $16 S$ rRNA gene have been applied to analyze various insect's gut microbiomes [20, 28-34].

The composition and diversity of the bacterial communities present in the gut of certain tephritids are not clearly illustrated. It has been observed that domestication, mass-rearing, diet, colony management and irradiation affect the insect gut microbiota $[8,20]$ which in turn impact the quality of mass-reared fruit flies used in SIT programs $[8,9,11,33]$, in addition, mass-reared fruit flies under laboratory conditions are not exposed to the natural microorganisms present in the environment. Hence, we studied the bacterial diversity and composition in midgut of wild as well as mass-reared $Z$. cucurbitae and $B$. dorsalis populations. We used Illumina HiSeq next-generation sequencing of $16 S$ rRNA gene to describe the bacterial communities in wild (male and female) and mass-reared mature ( $\sim 12$ days old) and newly emerged ( $<1 \mathrm{~h}$ after emergence) $Z$. cucurbitae and $B$. dorsalis male and female adults. We found that the gut bacterial communities associated with wild and mass-reared mature and newly emerged adults of $Z$. cucurbitae and $B$. dorsalis showed variation and depends on species and age of the insects.

\section{Results \\ 16S rRNA gene sequence reads}

We obtained an average of 122,227 high quality reads per sample in the variable regions of $\mathrm{V} 3$ and $\mathrm{V} 4$ region of $16 S$ rRNA gene. The total numbers of reads varied between wild male and female, also in mass-reared mature and newly emerged $Z$. cucurbitae and $B$. dorsalis adult samples. The Good's coverage was $99.9 \%$ in all samples of both fruit fly species indicating sequencing depth (Table 1). Rarefaction analysis showed that sequences sharply increased before approaching a plateau (Fig. 1).

\section{Bacterial diversity in gut of fruit fly species}

The bacterial richness and diversity was estimated for gut of wild and mass-reared Z. cucurbitae and B. dorsalis adults. Substantial diversity was observed in both stages of wild and mass-reared mature and newly emerged $Z$. cucurbitae and B. dorsalis adults, although coverage estimates were very high for all samples of fruit fly (Table 1). In case of $Z$. cucurbitae adults, the Shannon and Simpson indices of bacterial diversity showed difference for wild (Shannon diversity: 1.41-1.67 Simpson evenness: $0.29-0.34$ ), mass-reared mature (Shannon diversity: 1.05-1.31; Simpson evenness: 0.41-0.54) and newly emerged (Shannon diversity: 0.71-0.97; Simpson evenness: 0.62-0.69). Similarly, B. dorsalis wild (Shannon 
Table 1 Richness and diversity estimation of the 16S rRNA gene libraries from the metagenomic analysis of gut bacterial communities of wild and mass-reared Zeugodacus cucurbitae and Bactrocera dorsalis adult samples

\begin{tabular}{llll}
\hline $\begin{array}{l}\text { Fruit fly samples/ } \\
\text { Diversity index }\end{array}$ & $\begin{array}{l}\text { Shannon } \\
\text { diversity }\end{array}$ & $\begin{array}{l}\text { Simpson } \\
\text { evenness }\end{array}$ & $\begin{array}{l}\text { Goods } \\
\text { coverage (\%) }\end{array}$ \\
\hline WFC & 1.41 & 0.34 & 99.9 \\
WMC & 1.67 & 0.29 & 99.9 \\
WFD & 0.94 & 0.58 & 99.9 \\
WMD & 1.56 & 0.38 & 99.9 \\
MFC & 1.05 & 0.54 & 99.9 \\
MMC & 1.31 & 0.41 & 99.9 \\
NFC & 0.71 & 0.69 & 99.9 \\
NMC & 0.97 & 0.62 & 99.9 \\
MFD & 1.64 & 0.34 & 99.9 \\
MMD & 1.97 & 0.27 & 99.9 \\
NFD & 0.96 & 0.59 & 99.9 \\
NMD & 1.30 & 0.37 & 99.9 \\
\hline$Z$ C & &
\end{tabular}

$Z$ cucurbitae: WFC Wild female cucurbitae; WMC Wild male cucurbitae; MFC Mature female cucurbitae; MMC Mature male cucurbitae; NFC Newly emerged female cucurbitae; NMC Newly emerged male cucurbitae. B. dorsalis; WFD Wild female dorsalis; WMD Wild male dorsalis; MFD Mature female dorsalis; MMD Mature male dorsalis; NFD Newly emerged female dorsalis and NMD Newly emerged male dorsalis

diversity: 0.94-1.56; Simpson evenness: 0.38-0.58) and mass-reared mature (Shannon diversity: 1.64-1.97; Simpson evenness: 0.27-0.34) and newly emerged (Shannon diversity: 0.96-1.30; Simpson evenness: 0.37-0.59) adults were also showed variation in bacterial diversity (Table 1).
Relative abundance of bacterial taxa in the gut of fruit fly species

The relative abundance of bacterial phyla significantly varied between wild and mass-reared fruit fly species $(p<0.05)$ and age $(p<0.05)$ of adult samples. Five phyla Actinobacteria, Bacteroidetes, Cyanobacteria/Chloroplast, Firmicutes, and Proteobacteria were detected and there is significant difference $(p<0.05)$ in the samples of wild and mass-reared $Z$. cucurbitae and $B$. dorsalis adults (Fig. 2). Phylum Cyanobacteria/Chloroplast was present with very low relative abundance in the wild as well as in mass-reared fruit fly species. The Phylum Proteobacteria was more abundant in wild Z. cucurbitae $(\sim 87.72 \%)$ than wild $B$. dorsalis $(\sim 83.87 \%)$. Similarly, this phylum was also dominant in mass-reared $Z$. cucurbitae (64.15\%) and B. dorsalis ( 80.96\%) (Fig. 2). Among them, newly emerged fruit fly adults (83.85\%) contributed Proteobacteria which shows significantly higher prevalence than to mature fruit fly adults (61.25\%) (Fig. 2). The higher relative abundance of another phylum Firmicutes was observed in mass reared as compared to wild fruit fly adults. The bacterial communities belonging to Phylum Bacteroidetes was also observed in wild adults of both species in the range of $2.46-29.45 \%$. While range of relative abundance of this phylum was more in mature adults (7.08-22.08\%) than newly emerged (0.14-6.41\%) of Z. cucurbitae and B. dorsalis adults. Some reads were also recovered as unassigned bacteria from both the species samples (Fig. 2). Among fruit fly adults, sex of the insect did not have an effect

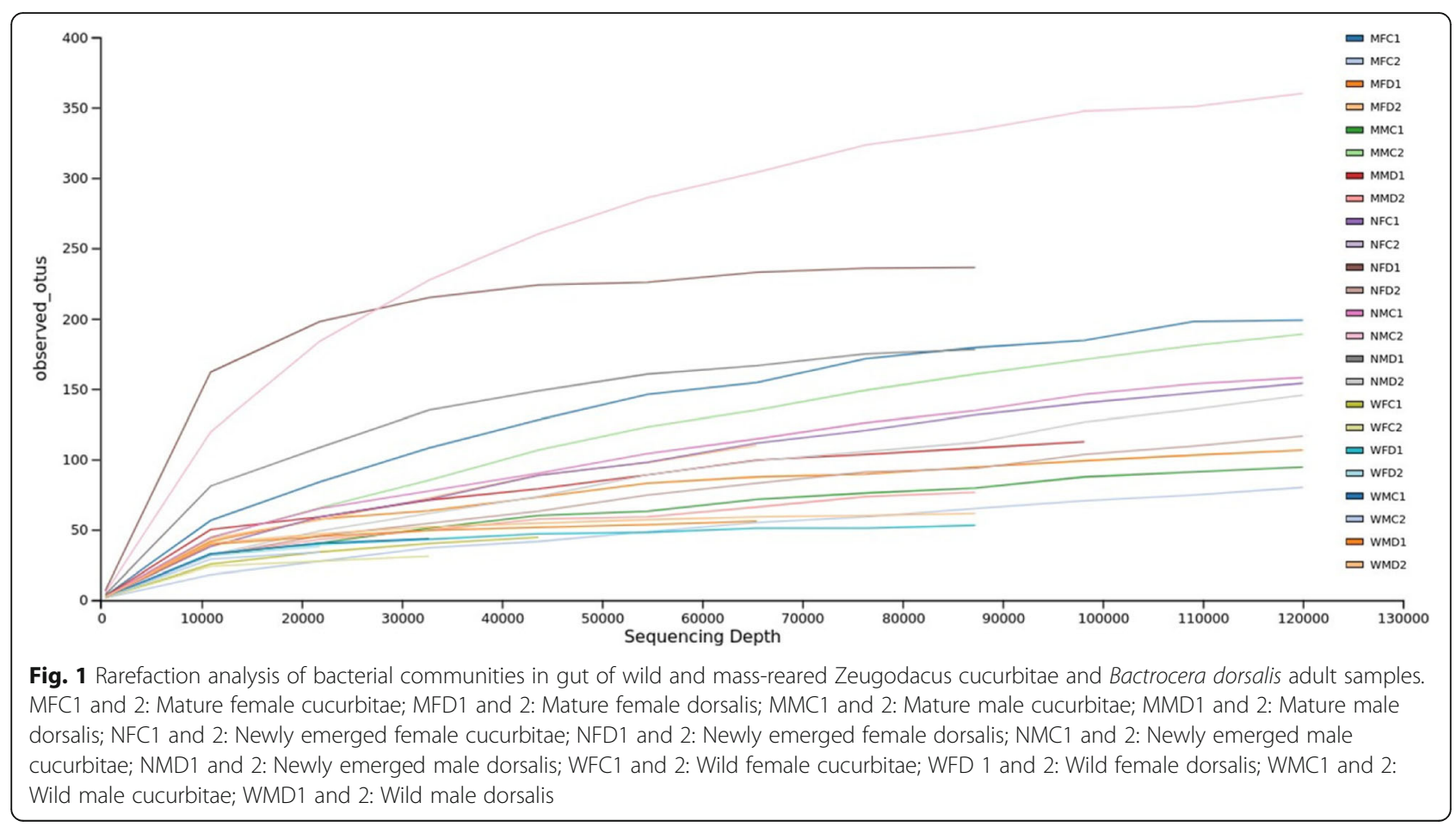




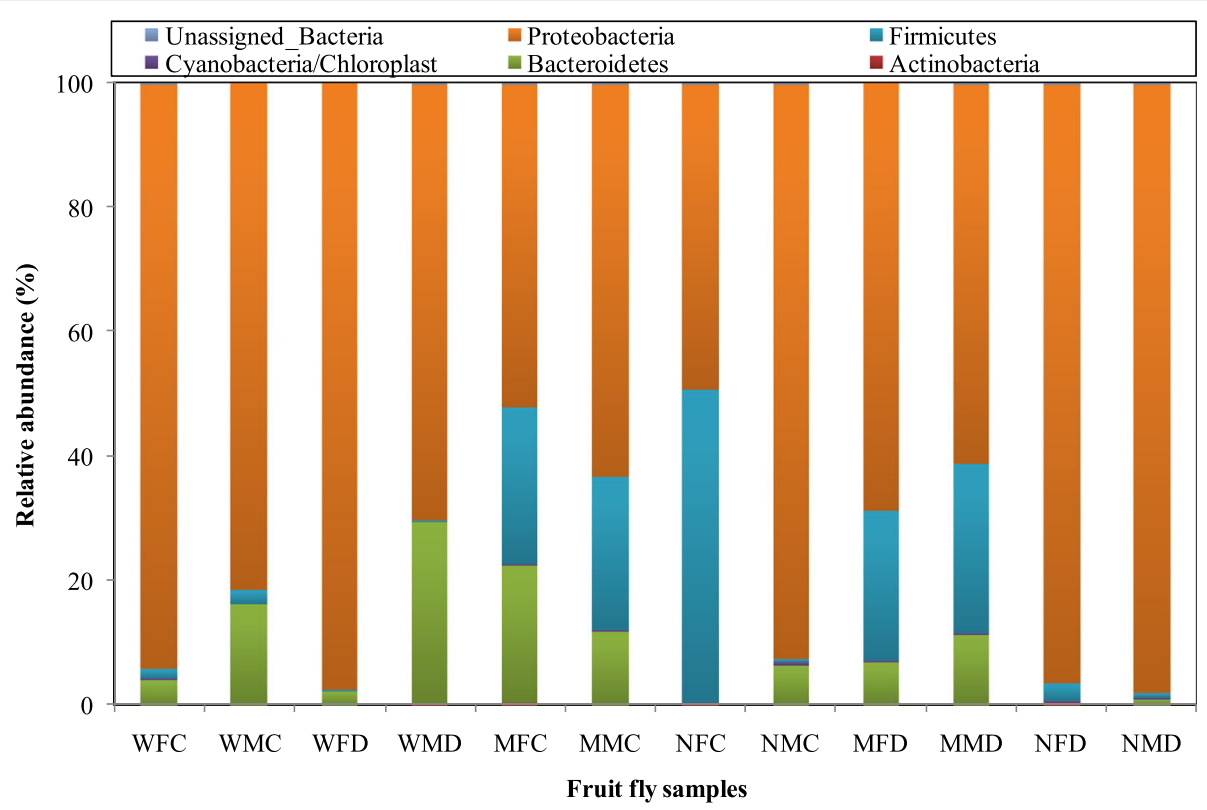

Fig. 2 Relative abundance (\%) of bacterial phyla obtained from the gut of wild and mass-reared mature and newly emerged Zeugodacus cucurbitae and Bactrocera dorsalis adult samples. Z. cucurbitae: WFC: Wild female cucurbitae; WMC: Wild male cucurbitae; MFC: Mature female cucurbitae; MMC: Mature male cucurbitae; NFC: Newly emerged female cucurbitae; NMC: Newly emerged male cucurbitae. B. dorsalis: WFD: Wild female dorsalis; WMD: Wild male dorsalis; MFD: Mature female dorsalis; MMD: Mature male dorsalis; NFD: Newly emerged female dorsalis and NMD: Newly emerged male dorsalis

on bacterial phyla composition $(p>0.05)$. The relative abundance and distribution of the bacteria from wild and mass-reared mature and newly emerged fruit fly species at class and order level are shown in Additional file 1: Figure S1 A \& B, respectively.

The bacterial families present in reads significantly varied with species $(p<0.05)$ and age of the of fruit fly adults $(p<0.05)$. Among the families, Enterobacteriaceae was detected in all samples of fruit fly species. This family was more predominant in wild $Z$. cucurbitae adults $(68.77 \%)$ as compared to mature (54.23\%) and newly emerged (46.22\%), whereas, Enterobacteriaceae was less abundant in wild B. dorsalis (53.22\%) than mature $(63.60 \%)$ and newly emerged (81.12\%) mass-reared adults (Fig. 3). Streptococcaceae and Flavobacteriaceae were other predominant families in wild as well as massreared Z. cucurbitae and B. dorsalis samples (Fig. 3). Pseudomonadaceae and Sphingobacteriaceae families were only detected in wild fruit fly adults of both species. Family Brucellaceae showed higher relative abundance in mass-reared newly emerged $Z$. cucurbitae as compared to wild and mass-reared matured adults. Moraxellaceae represented considerable level of relative abundance in newly emerged $B$. dorsalis than $Z$. cucurbitae. The relative abundance of Porphyromonadaceae family was more in mass-reared matured adults as compared to newly emerged adults.
Genus Providencia (Enterobacteriaceae) was present in wild as well as mass-reared fruit fly species adults. This genus was dominant in mass-reared newly emerged adults (ZC: 15.41\%; BD: 33.68\%) than mass-reared matured (ZC: $\sim 4.73 \%$; BD: $\sim 2.70 \%$ ) fruit fly samples (Fig. 4). Lactococcus is another genus frequently occurred in mass-reared fruit fly adults except newly emerged males of $Z$. cucurbitae samples (24.60\%). While, genus Acinetobacter was represented strongly in newly emerged male and females of both the species but occurred in low relative abundance in mature adults (Fig. 4). Other genera like Ochrobactrum, Myroides, Vagococcus, Corynebacterium, Staphylococcus and Enterococcus were also detected with lower relative abundance in both wild as well as in mass-reared fruit fly adults. While, Dysgonomonas and Wohlfahrtiimonas were only present in mass-reared fruit fly species adults (Fig. 4). Morganella and Serratia genera were also detected more in mass-reared mature and newly emerged $B$. dorsalis adults (Fig. 4). Overall, the bacterial genera showed a significant variation in relative abundance $(p<0.05)$ in wild and mass-reared fruit fly adults. The number of bacterial species and their relative abundance showed significant differences $(p<0.05)$ while no significant difference between fruit fly species $(p>0.05)$ and age of the insects $(p>0.05)$ was observed (Additional file 2: Table S1). The relative abundance of Empedobacter brevis, Myroides 


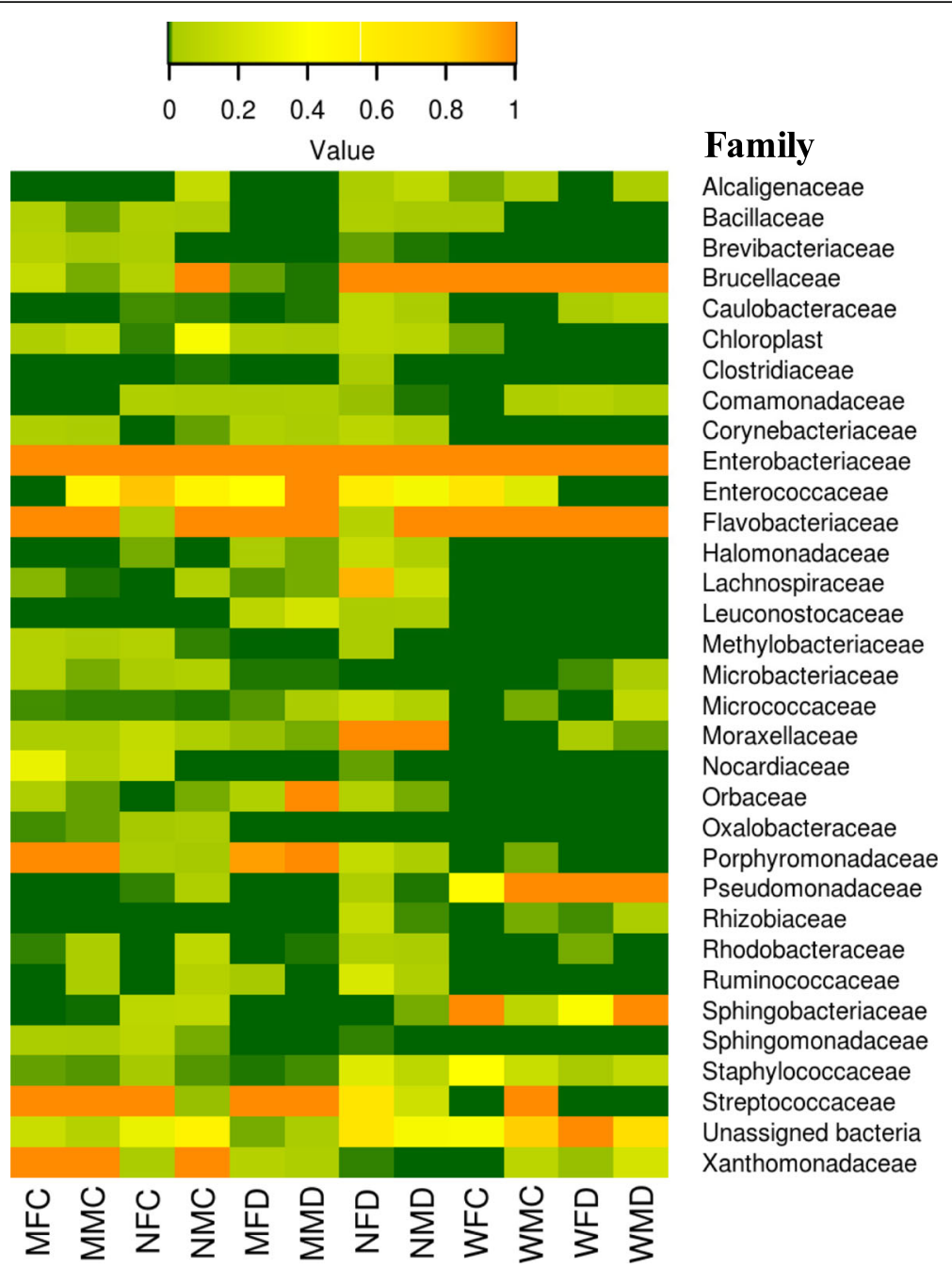

Fig. 3 Heat maps showing relative abundance of dominant bacterial families identified from gut of wild and mass-reared mature and newly emerged Zeugodacus cucurbitae and Bactrocera dorsalis adult samples. The color code indicates relative abundance, ranging from green (low abundance) to yellow to orange (high abundance). Z. cucurbitae: WFC: Wild female cucurbitae; WMC: Wild male cucurbitae; MFC: Mature female cucurbitae; MMC: Mature male cucurbitae; NFC: Newly emerged female cucurbitae; NMC: Newly emerged male cucurbitae. B. dorsalis: WFD: Wild female dorsalis; WMD: Wild male dorsalis; MFD: Mature female dorsalis; MMD: Mature male dorsalis; NFD: Newly emerged female dorsalis and NMD: Newly emerged male dorsalis

odoratus and Sphingobacterium yanglingense bacterial species were more prevalent in wild Z. cucurbitae and $B$. dorsalis adults (Additional file 2: Table S1). Myroides marinus, Dysgonomonas capnocytophagoides and Wohlfahrtiimonas larvae species were identified in mass-reared mature adults of both the species. While, Acinetobacter baumannii (8.9-15.7\%) occurred in mass-reared newly emerged B. dorsalis adults.

Overall relative abundance of bacterial communities present in the gut of wild as well as in mass-reared fruit fly species are significantly different. This indicates that the diet used during mass-rearing of insects significantly influences the bacterial taxa composition $(p<0.05)$. Sex of the insect did not have an effect on bacterial taxa $(p>0.05)$. Moreover, PCoA also confirmed that fruit fly adult samples were grouped based on fruit fly species and age of the adult i.e. wild and mass-reared (Fig. 5). However, no such grouping was formed on the basis of sex of the adult.

\section{Discussion}

The success of sterile insect technique (SIT) under field condition is greatly influenced by the fitness of sterile insects. The insect gut bacteria are known to diversified due to domestication, diet, colony management and irradiation $[8,20]$. Probiotic application of insect gut bacteria could enhance the fitness of sterile insects [7-11]. We used next generation sequencing technique to illustrate the gut bacterial composition of wild and massreared melon fly and Oriental fruit fly adults. The results 


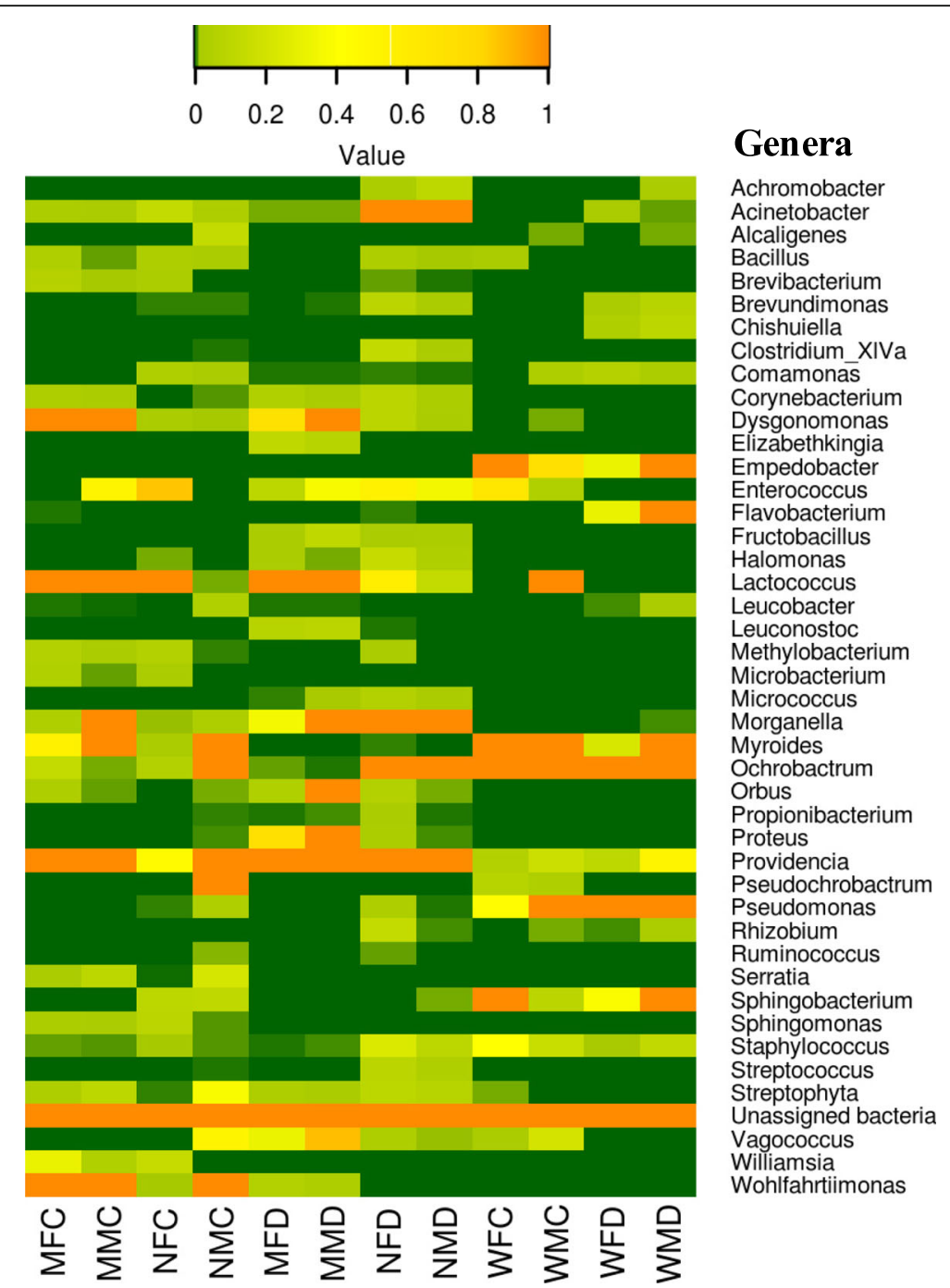

Fig. 4 Heat maps showing relative abundance of dominant bacterial genera identified from gut of wild and mass-reared mature and newly emerged Zeugodacus cucurbitae and Bactrocera dorsalis adult samples. The color code indicates relative abundance, ranging from green (low abundance) to yellow to orange (high abundance). Z. cucurbitae: WFC: Wild female cucurbitae; WMC: Wild male cucurbitae; MFC: Mature female cucurbitae; MMC: Mature male cucurbitae; NFC: Newly emerged female cucurbitae; NMC: Newly emerged male cucurbitae. B. dorsalis: WFD: Wild female dorsalis; WMD: Wild male dorsalis; MFD: Mature female dorsalis; MMD: Mature male dorsalis; NFD: Newly emerged female dorsalis and NMD: Newly emerged male dorsalis

indicate that significant difference was observed in relative abundance of bacterial communities in adults of $Z$. cucurbitae and B. dorsalis obtained from wild and massrearing facility. Four phyla Actinobacteria, Bacteroidetes, Firmicutes and Proteobacteria were occurred in all the samples of $Z$. cucurbitae and B. dorsalis adults. Phyla Actinobacteria, Bacteroidetes, Firmicutes and Proteobacteria are frequently recovered from different development stages of Bactrocera sp. [32, 34, 35]. Our results showed that Proteobacteria and Firmicutes are major phyla in wild as well as in mass-reared Z. cucurbitae and $B$. dorsalis adult samples. Proteobacteria was more represented in gut of wild as compared to mass-reared $Z$. cucurbitae and $B$. dorsalis adults. While, the relative abundance of Phylum Firmicutes was higher in mass reared insects as compared to wild fruit fly adults. It has been reported that these phyla are dominant in the gut of Bactrocera species adults like Chinese citrus fly (Bactrocera minax (Enderlein)), Carambola fruit fly (B. carambolae Drew \& Hancock) and B. dorsalis [32-34]. Actinobacteria and Bacteroidetes are also present in both Zeugodacus and Bactrocera sp. with varied relative abundance. Low level abundance of Bacteroidetes occurred in wild and mass reared mature $Z$. cucurbitae and $B$. dorsalis adults, while, Actinobacteria was observed only in newly emerged mass-reared adults. The occurrence of these phyla has been documented in $B$. dorsalis and B. carambolae [32-34]. It has been 


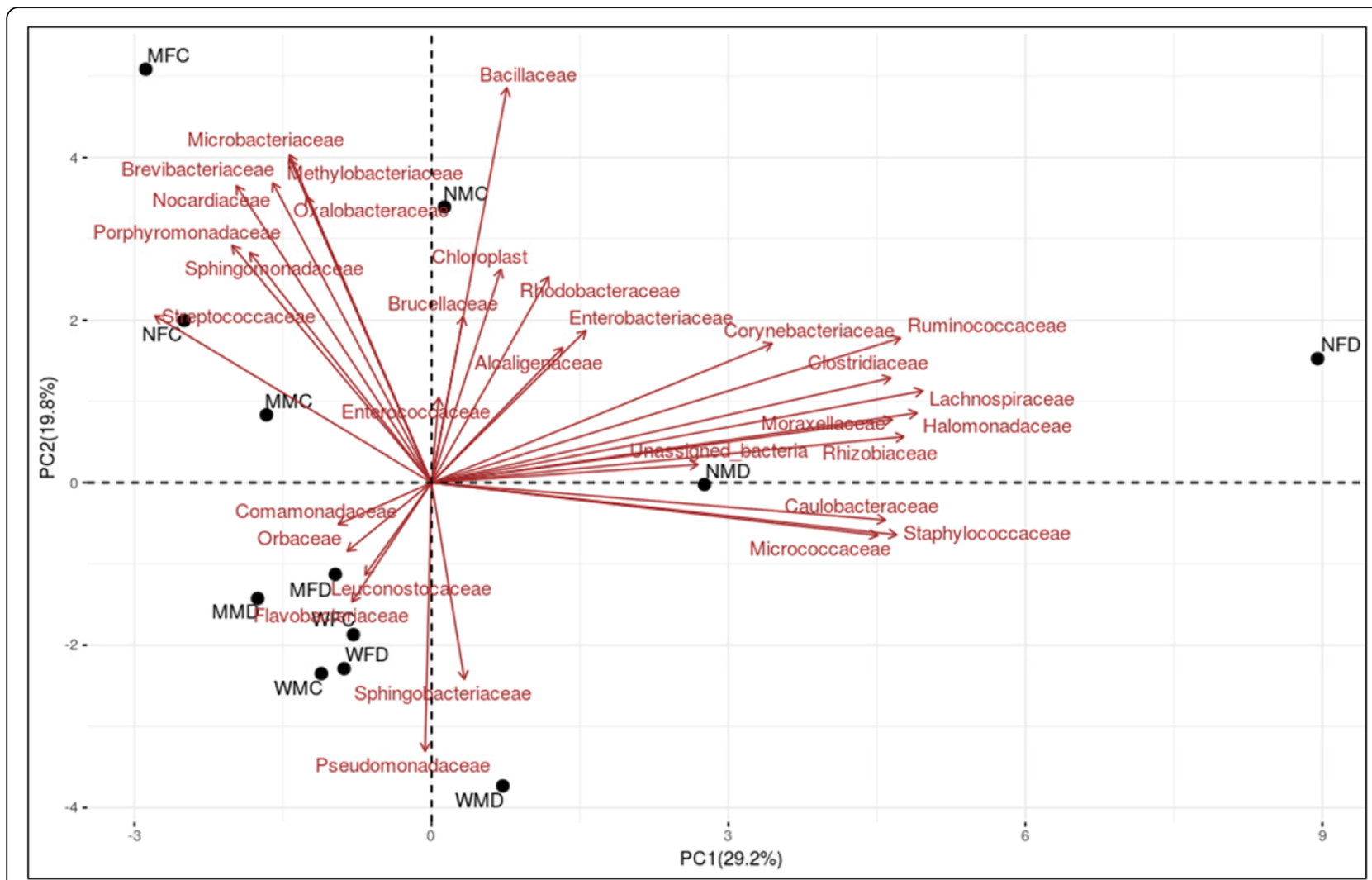

Fig. 5 Comparison of bacterial communities in wild and mass-reared Zeugodacus cucurbitae and Bactrocera dorsalis adult samples. Principal Coordinate Analysis (PCOA) was generated with bacterial composition of the families among wild and mass-reared fruit fly species. Z. cucurbitae: WFC: Wild female cucurbitae; WMC: Wild male cucurbitae; MFC: Mature female cucurbitae; MMC: Mature male cucurbitae; NFC: Newly emerged female cucurbitae; NMC: Newly emerged male cucurbitae. B. dorsalis: WFD: Wild female dorsalis; WMD: Wild male dorsalis; MFD: Mature female dorsalis; MMD: Mature male dorsalis; NFD: Newly emerged female dorsalis and NMD: Newly emerged male dorsalis

observed that Actinobacteria influences various metabolic and physiological activities including extracellular enzyme production, antimicrobial activity and other secondary metabolites formation [36, 37]. Thus, this phylum may be essential for contributing in growth and development of $Z$. cucurbitae and B. dorsalis.

In the present study, Enterobacteriaceae (ProteobacteriaGammaproteobacteria), Streptococcaceae (Firmicutes-Bacilli) and Flavobacteriaceae (Bacteroidetes-Flavobacteriia) were dominant families in both fruit fly species and the abundance varied with origin and age of the adults. Members of these families are known to be dominant and frequently identified from the gut of Bactrocera sp. [15-17, 19, 32-35]. The presence of Enterobacteriaceae in wild and mass-reared insects suggests important role of this family in insect growth and development [8].

Providencia (Enterobacteriaceae) occurred in wild as well as mass-reared adults followed by Lactococcus (Streptococcaceae) which is merely prevalent in wild fruit fly adults. This genus has been isolated from several fruit flies species including Anastrepha ludens (Loew), B. dorsalis, B. cucurbitae, B. minax and Bactrocera tau (Walker) [15-17, 23, 32, 33] and known to regulate the growth of other bacteria in the gut [38]. Other genera like Ochrobactrum, Myroides, Vagococcus, Corynebacterium, Staphylococcus and Enterococcus were observed in both wild as well as mass-reared fruit fly adults. This suggests the gut bacterial taxa significantly differ with fruit fly species, origin and age of the adults. Similarly, it has been also evident that different bacterial composition was observed among conspecific and interspecific B. carambolae and $B$. dorsalis samples through metagenomic analysis [34]. The deleterious microorganisms like Morganella and Serratia were detected only in mass-reared $Z$. cucurbitae and $B$. dorsalis adults. These genera are known to colonise in different mass-reared insects including fruit fly colonies $[25,32]$, this may be due to lower occurrence of certain members of Enterobacteriaceae which are known to prevent proliferation of pathogenic bacteria and also indirectly contributing in to host fitness $[8,11,12]$. Further, it has been observed that changes in the relative proportion of the 
different bacterial communities in the guts of mass reared medflies of the Vienna-8 strain [8].

The gut bacterial taxa significantly differed with the diet of fruit fly species. The specific natural diets used in the present study might have influenced the bacterial composition in wild and mass-reared fruit fly species and may play an important role in growth and development of insects. Some bacterial communities are more abundant in newly emerged adults as compared to wild and mass-reared mature adults. It has been observed that diet of insects and surroundings during mass rearing significantly influences the composition of gut microbiota $[8,11,20,39]$.

The influence of sex of the insects on the bacterial composition is minimal in both the fruit fly species in the present study. PCoA analysis also revealed that no groups were formed based on sexes of the species. Similarly, minimal variation in bacterial communities was observed in $B$. minax sexes, while significantly different bacterial communities were identified between the gut and the reproductive organs [32]. It has also been documented in medflies of the Vienna-8 strain that gut bacterial diversity varies with life stage and the sex of the insect, however, overall bacterial composition structure was not affected [11]. The present study showed the composition of bacterial communities in the gut of wild and mass-reared melon fly and Oriental fruit fly adults from India. The bacterial taxa were influenced by diet, varied with species and age but not with sex of the insects.

\section{Conclusions}

Gut bacterial diversity of wild and mass-reared Z. cucurbitae and $B$. dorsalis was analyzed using metagenomic sequencing of $16 S$ rRNA gene. Diversity of the inhabited bacterial communities from wild and mass-reared fruit fly adults was compared. Significant difference was observed in relative abundance of bacterial communities in the adults of $Z$. cucurbitae and B. dorsalis obtained from wild and mass-rearing facility. The most representative phyla were Actinobacteria, Bacteroidetes, Firmicutes and Proteobacteria in all samples of Z. cucurbitae and B. dorsalis adults. Among bacterial families, Enterobacteriaceae was predominant in the gut of wild and massreared adults of fruit fly. Genera Providencia and Lactococcus were dominant and present in all samples of the fruit fly with varied level of relative abundance. The PCoA analysis showed a distinct grouping of fruit fly species and age of the adults (wild, mature or newly emerged) while no grouping was formed based on the sex of the adults. Further studies will be focussed on identifying function of representative species for either pathogenic or probiotic activity of gut bacterial species. This will help in designing efficient mass rearing protocols for successful implementation of SIT in the field.

\section{Methods}

\section{Fruit fly sample collection}

The melon fly (Z. cucurbitae) infested pumpkin (Cucurbita sp.) were collected from cucurbits farm from Gouribidanur region of Karnataka state in India $\left(13^{\circ} 62^{\prime} \mathrm{N}\right.$ and $77^{\circ} 51^{\prime}$ E) while Oriental fruit fly (B. dorsalis) infested mango (Mangifera indica L.) were collected from Dahanu region of Maharashtra state in India $\left(19^{\circ} 97^{\prime} \mathrm{N}\right.$ and $72^{\circ}$ $\left.73^{\prime} \mathrm{E}\right)$. The infested fruits were brought to the laboratory and placed in sterile sand until pupation. Upon pupation, pupae were placed individually in sterile vials $(5 \mathrm{~mL}$ capacity) for adult emergence. These newly emerged adults of both the species are mentioned as 'wild' throughout the text. The wild adults emerged from pupae of both the species were used for gut extraction and further designated as follows: wild female $Z$. cucurbitae (WFC), wild male $Z$. cucurbitae (WMC) and wild female B. dorsalis (WFD) and wild male B. dorsalis (WMD) (Table 2). In case of laboratory reared (mass-reared), the melon fly ( $>36$ generations) and Oriental fruit fly (>30 generations) cultures were maintained on bottle gourd (Lagenaria siceraria (Molina) Standley) and sweet banana (Musa sp.) at $28 \pm 2{ }^{\circ} \mathrm{C}$ and $75 \% \mathrm{RH}$, respectively at insect rearing facility, Bhabha Atomic Research Centre (BARC), Mumbai, India. Adult flies were reared in two-side fine mesh cages $(45 \times 45 \times 45$ $\mathrm{cm}$ ) and provided with ProtineX (Nutricia International Private Ltd., Mumbai, India) and water. Upon pupation, pupae were placed individually in sterile vials $(5 \mathrm{~mL}$ capacity) for adult emergence. The mature ( 12 days old $)$ (M) and newly emerged (N) $(<1 \mathrm{~h}$ after emergence) male and female adults of $Z$. cucurbitae and B. dorsalis were used for gut extraction and further designated as follows: mature female of $Z$. cucurbitae (MFC), mature male of $Z$. cucurbitae (MMC), newly emerged female of $Z$. $\underline{\text { cucurbi- }}$ tae (NFC); newly emerged male of $\bar{Z}$. cucurbitae (NMC); mature female of $B$. dorsalis (MFD); mature male of $B$. $\overline{\text { dorsalis }}^{-}$(MMD); newly emerged female of $\bar{B}$. dorsalis (NFD) and newly emerged male of $B$. dorsalis (NMD) (Table 2).

\section{Fruit fly dissection and DNA extraction}

The wild and mass-reared male and female adults of melon fly and Oriental fruit fly were surface sterilized by submerging them sequentially in $70 \%$ ethanol for $1 \mathrm{~min}$, $0.5 \%$ sodium hypochlorite for $1 \mathrm{~min}$ and washed twice (1 min each) in sterile distilled water [16, 19]. Total thirty male and female of wild and mass-reared adults of both species were used in this study. The surface sterilized flies were individually dissected $(n=15$ of each adult species/replicate; two replicates) aseptically under a clean air workstation. The insect midgut portion extending from the crop to the joints of malpighian tubules were separated and transferred to a sterile $1.5 \mathrm{~mL}$ microcentrifuge tube. Total genomic DNA was extracted 
Table 2 The melon fly (Zeugodacus cucurbitae) and Oriental fruit fly (Bactrocera dorsalis) adult populations used in this study

\begin{tabular}{lllll}
\hline Wild or mass-reared adults & Accession code & Host & Location & Number of samples $^{\text {a }}$ \\
\hline Z. cucurbitae & WFC & Cucurbita sp. & Gouribidanur, Karnataka, India & 30 \\
& WMC & Cucurbita sp. & Gouribidanur, Karnataka, India & 30 \\
B. dorsalis & WFD & Mangifera indica & Dahanu, Maharashtra, India & 30 \\
& WMD & Mangifera indica & Dahanu, Maharashtra, India & 30 \\
Z. cucurbitae & Lagenaria siceraria & Insect rearing facility, BARC, Mumbai, India & 30 \\
& MMC & Lagenaria siceraria & Insect rearing facility, BARC, Mumbai, India & 30 \\
& NFC & Lagenaria siceraria & Insect rearing facility, BARC, Mumbai, India & 30 \\
B. dorsalis & Lagenaria siceraria & Insect rearing facility, BARC, Mumbai, India & 30 \\
& MFD & Musa sp. & Insect rearing facility, BARC, Mumbai, India & 30 \\
& MMD & Musa sp. & Insect rearing facility, BARC, Mumbai, India & 30 \\
& NFD & Insect rearing facility, BARC, Mumbai, India & 30 \\
\hline
\end{tabular}

Z cucurbitae: WFC Wild female cucurbitae; WMC Wild male cucurbitae; MFC Mature female cucurbitae; MMC Mature male cucurbitae; NFC Newly emerged female cucurbitae; NMC Newly emerged male cucurbitae. B. dorsalis; WFD Wild female dorsalis; WMD Wild male dorsalis; MFD Mature female dorsalis; MMD Mature male dorsalis; NFD Newly emerged female dorsalis and NMD: Newly emerged male dorsalis.

${ }^{\text {a }}$ Pooled DNA of fifteen adult samples of male/female as one replicate and two replicates were used for both species in this study

from the pooled gut content obtained from $Z$. cucurbitae and $B$. dorsalis samples by using PureLink Genomic DNA kit (Invitrogen, Germany) according to manufacturer's instructions. The quantity and quality of DNA was determined by NanoDrop 2000 (Thermo Scientific, USA) spectrophotometric analysis at $260 \mathrm{~nm}$ and agarose gel $(0.8 \%)$ electrophoresis.

\section{PCR, Illumina sequencing and data processing}

Bacterial communities present in the gut of wild and mass-reared adults of $Z$. cucurbitae and B. dorsalis were studied using Illumina HiSeq next-generation sequencing (NGS) of $16 S$ rRNA gene. Polymerase chain reaction (PCR) was performed as described with PCR Protocol for Phusion ${ }^{\circ}$ High-Fidelity DNA Polymerase (New England Biolabs, UK). We used Pro341F (5' - CCTACGGG NBGCASCAG-3') and Pro805R (5'-GACTACNVGGGTATCTAATCC-3') primers to amplify the V3-V4 regions of the $16 S$ rRNA gene [40]. PCR products were further purified using PureLink ${ }^{\circ}$ PCR Purification Kit (Invitrogen, Germany). Sequencing was conducted using a paired-end, $2 \times 250$-bp cycle run on an Illumina HiSeq Rapid V2 kit (AgriGenome Labs Pvt. Ltd., Kochi, India).

Demultiplexed raw sequences were extracted from the Illumina HiSeq system in FASTQ format and the quality of sequences was evaluated using the bcl2fastQ software (Illumina Inc). The reads were pre-processed in UPARSE implemented in USEARCH 10.0.240 [41]. We have used the maximum overlap $150 \mathrm{bp}$ and minimum overlap of $30 \mathrm{bp}$ to get all possible consensuses. While making consensus V3-V4 sequences, the passed reads were further aligned to each other with a contig length of 400-480 bp. The first step was merging paired reads of all samples into a single FASTQ file. The primers were stripped and the filtered sequences were obtained. The reads with $>$ Q30 were included in the analysis. Further, singletons were discarded and unique and abundant sequences identified using USEARCH [42]. Chimeras were removed using cluster_otus command implemented in the tool USEARCH [37]. The sequences were subsequently clustered into operational taxonomic units (OTUs) at a 97\% similarity level. Representative sequence and taxonomy classification was identified for each OTU and aligned against the Ribosomal Database Project (RDP) training set of sequences (http://www.drive5.com/sintax/rdp_16s_ v16_sp.fa.gz). QIIME program (QIIME 2-2018.8) was employed for the further downstream analysis by using OTU table after converting to the Biological Observation Matrix (BIOM) format [43]. The alpha diversity indices and rarefaction analysis were obtained using QIIME (22018.8). Further, heat maps [44] were generated from relative abundance of family and genera while Principal Coordinate Analysis (PCoA) plot was obtained from bacterial composition of families implemented in $\mathrm{R}$ program ver. 3.5. (https://www.R-project.org/). We used nonparametric Kruskal-Wallis tests in R v. 3.4.1 to determine whether there were significant differences in the relative abundances of bacterial taxa (phylum to species) in fruit fly adult samples. We selected all the bacterial taxa which were contributing at least $>0.01 \%$ of relative abundance in any one of study groups.

\section{Supplementary information}

Supplementary information accompanies this paper at https://doi.org/10. 1186/s12866-019-1647-8.

Additional file 1: Figure S1. Relative abundance (\%) of major bacterial classes (A) and orders (B) identified in the gut of wild and mass-reared 
Zeugodacus cucurbitae and Bactrocera dorsalis adult samples revealed by metagenomic analysis. Z. cucurbitae: WFC: Wild female cucurbitae; WMC: Wild male cucurbitae; MFC: Mature female cucurbitae; MMC: Mature male cucurbitae; NFC: Newly emerged female cucurbitae; NMC: Newly emerged male cucurbitae. B. dorsalis: WFD: Wild female dorsalis; WMD: Wild male dorsalis; MFD: Mature female dorsalis; MMD: Mature male dorsalis; NFD: Newly emerged female dorsalis and NMD: Newly emerged male dorsalis.

Additional file 2: Table S1. Relative abundance (\%) of major bacterial species (with $>1 \%$ in at least one adult sample) in gut of wild and massreared Zeugodacus cucurbitae and Bactrocera dorsalis adult samples.

\section{Abbreviations}

BD: Bactrocera dorsalis; BIOM: Biological Observation Matrix;

DGGE: Denaturing gradient gel electrophoresis; NGS: Next-generation sequencing; OTU: Operational taxonomic units; PCOA: Principal Coordinate Analysis; SIT: Sterile insect technique; ZC: Zeugodacus cucurbitae

\section{Acknowledgements}

We thank Dr. V. P. Venugopalan, Associate Director, Bioscience Group and Head, NA\&BTD, BARC, Mumbai. We appreciate Mr. Vikas and Mr. S. M. Tota for help in mass-rearing of fruit fly species.

\section{About this supplement}

This article has been published as part of BMC Microbiology Volume 19 Supplement 1, 2019: Proceedings of an FAO/IAEA Coordinated Research Project on Use of Symbiotic Bacteria to Reduce Mass-rearing Costs and Increase Mating Success in Selected Fruit Pests in Support of SIT Application: microbiology. The full contents of the supplement are available online at https://bmcmicrobiol. biomedcentral.com/articles/supplements/volume-19-supplement-1.

\section{Authors' contributions}

Conceived and designed the study: $\mathrm{ABH}, \mathrm{RSH}$. Performed the experiments: $\mathrm{ABH}, \mathrm{RSH}$. Analyzed the sequence data: SKGS, ABH, RSH. Wrote the paper: $A B H, R S H, S K G S$. All authors read and approved the final manuscript.

\section{Funding}

This research was conducted under the Coordinated Research Project (Research contract no. 17090) supported by International Atomic Energy Agency (IAEA), Vienna.

\section{Availability of data and materials}

The data that support the findings of this study are available from the corresponding author upon reasonable request.

\section{Ethics approval and consent to participate}

Not applicable.

\section{Consent for publication}

Not applicable.

\section{Competing interests}

The authors declare that they have no competing interest.

\section{Author details}

${ }^{1}$ Nuclear Agriculture \& Biotechnology Division, Bhabha Atomic Research Centre, Trombay, Mumbai 400 085, India. ${ }^{2}$ Cytogenetics and Molecular Genetics Section, Pathology Unit, Medical Division, Bhabha Atomic Research Centre, Trombay, Mumbai 400 085, India. ${ }^{3} \mathrm{Homi}$ Bhabha National Institute (HBNI), Training School Complex, Anushaktinagar, Mumbai 400 094, India.

\section{Published: 24 December 2019}

\section{References}

1. White IM, Elson-Harris MM. Fruit flies of economic significance: their identification and bionomics. Wallingford: CAB International; 1992. p. 601.

2. Dhillon MK, Singh R, Naresh JS, Sharma HC. The melon fruit fly, Bactrocera cucurbitae: A review of its biology and management. J Insect Sci. 2005;5:40.
3. Virgilio M, Delatte H, Backeljau T, De Meyer M. Macrogeographic population structuring in the cosmopolitan agricultural pest Bactrocera cucurbitae (Diptera:Tephritidae). Mol Ecol. 2010;19:2713-24.

4. Verghese A, Madhura HS, Jayanthi PDK, Stonehouse JM. Fruit flies of economic significance in India with special reference to Bactrocera dorsalis Hendel. In: Barnes BN, editor. Proceedings of the sixth international symposium on fruit flies of economic importance. Stellenbosch: Isteg Scientific Publications; 2004. p. 317-24.

5. Koyama J, Kakinohana H, Miyatake T. Eradication of the melon fly, Bactrocera cucurbitae in Japan: importance of behavior, ecology, genetics, and evolution. Annu Rev Entomol. 2004:49:331-49.

6. Dyck VA, Hendrichs J, Robinson AS. Sterile insect technique: principles and practice in area-wide integrated Pest management. Dordrecht: Springer; 2005.

7. Niyazi N, Lauzon CR, Shelly TE. Effect of probiotic adult diets on fitness components of sterile male Mediterranean fruit flies (Diptera: Tephritidae) under laboratory and field cage conditions. J. Eco. Entomol. 2004;97:1570-80.

8. Ben-Ami E, Yuval B, Jurkevitch E. Manipulation of the microbiota of mass reared Mediterranean fruit flies Ceratitis capitata (Diptera: Tephritidae) improves sterile male sexual performance. ISME J. 2010;4:28-37.

9. Gavriel S, Gazit Y, Jurkevitch E, Yuval B. Bacterially enriched diet improves sexual performance of sterile male Mediterranean fruit flies. J Appl Entomol. 2011;135:564-73.

10. Hamden H, Guerfali MM, Fadhl S, Saidi M, Chevrier C. Fitness improvement of mass-reared sterile males of Ceratitis capitata (Vienna 8 strain) (Diptera: Tephritidae) after gut enrichment with probiotics. J Eco Entomol. 2013;106:641-7.

11. Augustinos AA, Kyritsis GA, Papadopoulos NT, Abd-Alla AMM, Cáceres C, Bourtzis K. Exploitation of the medfly gut microbiota for the enhancement of sterile insect technique: Use of Enterobacter sp in larval diet-based probiotic applications. PLoS ONE. 2015;10:e0136459.

12. Dillon RJ, Dillon VM. The gut bacteria of insects: nonpathogenic interactions. Annu Rev Entomol. 2004:49:71-92.

13. Engel $P$, Moran NA. The gut microbiota of insects - diversity in structure and function. FEMS Microbiol Rev. 2013;37:699-735.

14. Douglas AE. The multi-organismal insect: diversity and function of resident microorganisms. Annu Rev Entomol. 2015;60:17-34.

15. Prabhakar CS, Sood P, Kanwar SS, Sharma PN, Kumar A, Mehta PK. Isolation and characterization of gut bacteria of fruit fly, Bactrocera tau (Walker) (Diptera: Tephritidae). Phytoparasitica. 2012;41:193-201.

16. Thaochan N, Drew RAl, Hughes JM, Vijaysegaran S, Chinajariyawong A. Alimentary tract bacteria isolated and identified with API-20E and molecular cloning techniques from Australian tropical fruit flies, Bactrocera cacuminata and B. tryoni. J. Insect Sci. 2010;10:1-16.

17. Wang $H$, Jin $L$, Zhang $H$. Comparison of the diversity of the bacterial communities in the intestinal tract of adult Bactrocera dorsalis from three different populations. J Appl Microbiol. 2011;110:1390-401.

18. Wang $H$, Jin L, Peng $T$, Zhang $H$, Chen $Q$, Hua $Y$. Identification of cultivable bacteria in the intestinal tract of Bactrocera dorsalis from three different populations and determination of their attractive potential. Pest Manag Sci. 2013;70:80-7.

19. Hadapad AB, Prabhakar CS, Chandekar SC, Tripathi J, Hire RS. Diversity of bacterial communities in the midgut of Bactrocera cucurbitae (Diptera: Tephritidae) populations and their potential use as attractants. Pest Manag Sci. 2016:72:1222-30

20. Deutscher AT, Burke CM, Darling AE, Riegler M, Reynolds OL, Chapman TA. Near full-length 165 rRNA gene next-generation sequencing revealed Asaia as a common midgut bacterium of wild and domesticated Queensland fruit fly larvae. Microbiome. 2018;6:85.

21. Martinez AJ, Robacker DC, Garcia JA, Esau KL. Laboratory and field olfactory attraction of the Mexican fruit fly (Diptera: Tephritidae) to metabolites of bacterial species. Fla Entomol. 1994;77:117-26.

22. Sood P, Prabhakar CS, Mehta PK. Eco-friendly management of fruit flies through their gut bacteria. J Insect Sci. 2010;23:275-83.

23. Kuzina LV, Peloquin JJ, Vacek DC, Miller TA. Isolation and identification of bacteria associated with adult laboratory Mexican fruit flies, Anastrepha ludens (Diptera: Tephritidae). Curr Microbiol. 2001;42:290-4.

24. Aharon Y, Pasternak Z, Ben Yosef M, Behar A, Lauzon C, Yuval B, Jurkevitch E. Phylogenetic, metabolic, and taxonomic diversities shape Mediterranean fruit fly microbiotas during ontogeny. Appl Environ Microbiol. 2013;79:303-13.

25. Pramanik MM, Mahin AA, Khan M, Miah AB. Isolation and identification of mid-gut bacterial community of Bactrocera dorsalis (Hendel) (Diptera: Tephritidae). Res J Microbiol. 2014;9:278-86. 
26. Brauman A, Dore J, Eggleton P, Bignell D, Breznak JA, Kane MD. Molecular phylogenetic profiling of prokaryotic communities in guts of termites with different feeding habits. FEMS Microbiol Ecol. 2001;35:27-36.

27. Rani A, Sharma A, Rajagopal R, Adak T, Bhatnagar RK. Bacterial diversity analysis of larvae and adult midgut microflora using culture-dependent and culture-independent methods in lab-reared and field-collected Anopheles stephensi - an Asian malarial vector. BMC Microbiol. 2009;9:96.

28. Warnecke F, Luginbühl P, Ivanova N, Ghassemian M, Richardson TH, Stege JT, Cayouette M, McHardy AC, Djordjevic G, Aboushadi N. Metagenomic and functional analysis of hindgut microbiota of a wood-feeding higher termite. Nature. 2007:450:560-5.

29. Minard G, Tran FH, Dubost A, Tran-Van V, Mavingui P, Moro CV. Pyrosequencing $16 \mathrm{~S}$ rRNA genes of bacteria associated with wild tiger mosquito Aedes albopictus: a pilot study. Front Cell Infect Microbiol. 2014;4:59.

30. Cox-Foster DL, Conlan S, Holmes EC, Palacios G, Evans JD, Moran NA, Quan $P$, Briese T, Hornig M, Geiser DM. A metagenomic survey of microbes in honey bee colony collapse disorder. Science. 2007;318:283-7.

31. Ishak HD, Plowes R, Sen R, Kellner K, Meyer E, Estrada DA, Dowd SE, Mueller UG. Bacterial diversity in Solenopsis invicta and Solenopsis geminata ant colonies characterized by 165 amplicon 454 pyrosequencing. Microb Ecol. 2011;61:821-31.

32. Wang A, Yao Z, Zheng W, Zhang H. Bacterial communities in the gut and reproductive organs of Bactrocera minax (Diptera: Tephritidae) based on 454 pyrosequencing. PLoS One. 2014;9:e106988.

33. Andongma AA, Wan L, Dong YC, Li P, Desneux N, White JA, Niu CY. Pyrosequencing reveals a shift in symbiotic bacteria populations across life stages of Bactrocera dorsalis. Sci Rep. 2015;5:9470.

34. Yong HS, Song SL, Chua KO, Lim PE. Microbiota associated with Bactrocera carambolae and B. dorsalis (Insecta: Tephritidae) revealed by next-generation sequencing of 16S rRNA gene. Meta Gene. 2017;11:189-96.

35. Morrow JL, Frommer M, Sherman DCA, Riegler M. The microbiome of field caught and laboratory-adapted Australian tephritid fruit fly species with different host plant use and specialisation. Microb Ecol. 2015;70:498-508.

36. Schrempf H. Recognition and degradation of chitin by Streptomycetes. Antonie Van Leeuwenhoek. 2001;79:285-9.

37. Zhao X, Zhang X, Chen Z, Wang Z, Lu Y, Cheng D. The divergence in bacterial components associated with Bactrocera dorsalis across developmental stages. Front Microbiol. 2018;9:114.

38. Fooks $\sqcup$, Gibson GR. (2002) probiotics as modulators of the gut flora. Br J Nutr. 2002;88:S39-49.

39. Colman DR, Toolson EC, Takacs VCD. Do diet and taxonomy influence insect gut bacterial communities? Mol Ecol. 2012;21:5124-37.

40. Takahashi S, Tomita J, Nishioka K, Hisada T, Nishijima M. Development of a prokaryotic universal primer for simultaneous analysis of bacteria and archaea using next-generation sequencing. PLoS One. 2014;9:e105592.

41. Edgar RC. UPARSE: highly accurate OTU sequences from microbial amplicon reads. Nat Methods. 2013;10:996-8.

42. Edgar RC, Haas BJ, Clemente JC, Quince C, Knight R. UCHIME improves sensitivity and speed of chimera detection. Bioinformatics. 2011;27:2194-200.

43. McDonald D, Clemente JC, Kuczynski J, Rideout JR, Stombaugh J, Wendel D, Wilke A, Huse S, Hufnagle J, Meyer JF, Knight R, Caporaso JG. The biological observation matrix (BIOM) format or: how I learned to stop worrying and love the ome-ome. GigaScience. 2012;1:7.

44. Warnes GR, Bolker B, Bonebakker L, Gentleman R, Huber W, Liaw A, Lumley T, Maechler M, Magnusson A, Moeller S, Schwartz M, Venables B. gplots: Various R Programming Tools for Plotting Data. R package version 3.0.1. 2016. https://CRAN.R-project.org/package=gplots.

\section{Publisher's Note}

Springer Nature remains neutral with regard to jurisdictional claims in published maps and institutional affiliations.

Ready to submit your research? Choose BMC and benefit from:

- fast, convenient online submission

- thorough peer review by experienced researchers in your field

- rapid publication on acceptance

- support for research data, including large and complex data types

- gold Open Access which fosters wider collaboration and increased citations

- maximum visibility for your research: over $100 \mathrm{M}$ website views per year

At BMC, research is always in progress.

Learn more biomedcentral.com/submissions 